\section{ARTIGO ORIGINAL ORIGINAL ARTICLE}

\title{
Aedes aegypti e sociedade: o impacto econômico das arboviroses no Brasil
}

\author{
Aedes aegypti and society: the economic \\ burden of arboviruses in Brazil \\ Vanessa Teich', Roberta Arinelli², Lucas Fahham³ \\ DOI: 10.21115/JBES.v9.n3.p267-76
}

\section{RESUMO}

Objetivo: Arboviroses são motivo de preocupação para a saúde pública, com impactos clínicos e

\section{Palavras-chave:}

dengue, infecção pelo Zika vírus, febre de chikungunya, custos e análise de custo, infecções por arbovírus

\section{Keywords:}

dengue, zika virus infection, chikungunya fever, costs and cost analysis, arbovirus infections

\begin{abstract}
Objective: Arboviruses are a cause of concern for public health, with negative clinical and economic impacts. The objective of this study was to evaluate costs of vector control, direct medical costs and indirect costs associated with classical dengue fever, dengue hemorrhagic fever, chikungunya and Zika virus infection in Brazil for the year 2016. Methods: The analyzes quantified costs related to vector control, such as federal resource transfers and acquisition of pesticides, direct medical costs related to diseases treatment through outpatient and hospital management, and indirect costs related to absenteeism, calculated from published studies or estimates of disability-adjusted life years (DALYs). Results: The investment for vector combat was R\$1.5 billion in Brazil and the cost reported by federal government for the acquisition of insecticides and larvicides was R\$ 78.6 million. Direct medical costs generated total expenses of $\mathrm{R} \$ 374$ million. Fever chikungunya presented the highest number of DALYs lost per episode of disease (0.036 DALYs), followed by Zika virus infection (0.005 DALYs). Total indirect cost was estimated at R\$ 431 million. Total costs with the management of arboviroses reached an impact of R\$2.3 billion in Brazil for 2016. Minas Gerais, São Paulo, Bahia and Rio de Janeiro presented the highest costs. Conclusions: Arboviroses generate considerable economic and social impact to Brazil. Vector combat costs, direct medical costs and indirect costs account for about 2\% of the health budget expected for the country in 2016.
\end{abstract}

Recebido em: 15/01/2018. Aprovado para publicação em: 26/02/2018

1. Vice-presidente, Sense Company, São Paulo, SP, Brasil.

2. Diretora médica, Sense Company, Rio de Janeiro, RJ, Brasil.

3. Gerente de Projetos, Sense Company, São Paulo, SP, Brasil.

Instituição onde o trabalho foi executado: Sense Company, São Paulo, SP, Brasil.

Informações sobre auxílios recebidos sob a forma de financiamento, equipamentos ou medicamentos: Este estudo foi financiado pela Oxitec Brasil.

Autor correspondente: Vanessa Teich, Av. Paulista, 1.374, 11andar, São Paulo/SP, Brasil. E-mail: vanessa.teich@wearesense. company. 


\section{Introdução}

Arbovírus incluem os vírus da febre amarela (YFV), dengue (DENV), chikungunya (CHIKV) e Zika (ZIKV), entre outros, e têm sido motivo de grande preocupação para a saúde pública em todo o mundo (Donalisio et al., 2017). As manifestações clínicas de infecções por arbovírus podem variar de doença febril leve a síndromes febris hemorrágicas, articulares e neurológicas. De forma geral, os quadros graves são detectados após circulação viral em extensas epidemias, com impactos imprevisíveis na morbidade e na mortalidade (Donalisio et al., 2017).

Nos últimos anos, a incidência de doenças causadas por arbovírus apresentou um aumento global relevante (Gould et al., 2017), que está correlacionado a fatores como dispersão mais rápida e geograficamente mais extensiva dos vírus em razão do crescimento intensivo dos sistemas de transporte globais, adaptação dos vetores à urbanização crescente, incapacidade de conter a população de mosquitos e alterações em fatores ambientais (Gould et al., 2017). Além dos fatores que favorecem a dispersão das doenças, o Brasil representa um país com condições ambientais ótimas para a permanência e disseminação de mosquitos vetores, como o Aedes aegypti (Gregianini et al., 2017).

Além do impacto clínico negativo, o fardo econômico associado às arboviroses é preocupante. Apesar de a maioria dos pacientes apresentar recuperação completa após a fase aguda da doença, alguns sintomas podem durar semanas ou meses, interferindo nas atividades laborais, e algumas síndromes podem cursar com incapacidades permanentes (Donalisio et al., 2017; Ministério da Saúde [Brasil], 2016a; Ministério da Saúde [Brasil], 2017a).

Nas últimas décadas, houve a disseminação da dengue nas Américas. Em 2015, mais de dois milhões de casos dessa doença foram notificados e, entre eles, 1,65 milhão foram registrados no Brasil, com 811 óbitos e taxa de incidência de 813 casos por 100 mil habitantes (Donalisio et al., 2017; Ministério da Saúde [Brasil], 2016b). O custo associado ao manejo da dengue no Brasil é considerado o maior das Américas, correspondendo a $42 \%$ dos gastos totais relacionados à doença no continente (Shepard et al., 2011). Além disso, entre todos os países do hemisfério ocidental, o Brasil apresentou os maiores gastos anuais agregados induzidos pela dengue, para o período de 2000 a 2007, com média de US\$ 1,35 bilhão/ano, quando considerados custos diretos médicos e não médicos e custos indiretos decorrentes da perda de produtividade (Shepard et al., 2011).

As primeiras infecções autóctones por CHIKV no Brasil ocorreram em 2014, nas regiões Norte e Nordeste (Nunes et al., 2015). Em 2015, 38.499 casos prováveis de febre de chikungunya foram registrados no Brasil e, em 2016, 271.824 casos (Ministério da Saúde [Brasil], 2017b). Os sinais e os sintomas são clinicamente parecidos com os da dengue e a principal manifestação clínica que as difere são as dores articulares intensas. Após a fase inicial, a doença pode evoluir para as fases subaguda e crônica, com duração de até três anos (Ministério da Saúde [Brasil], 2017a). Embora não esteja relacionada à alta letalidade, cursa com elevada taxa de morbidade pela artralgia persistente e destrutiva em alguns casos, tendo como consequências perda de produtividade e redução da qualidade de vida relacionada à saúde, além de seus custos associados (Ministério da Saúde [Brasil], 2017a).

Em 2016, 215.319 casos prováveis de febre por ZIKV foram reportados no país, com incidência de 105,3 casos por 100 mil habitantes (Ministério da Saúde [Brasil], 2017b). A infecção clássica por ZIKV cursa como uma doença febril autolimitada, com duração de três a seis dias e sintomas como conjuntivite, cefaleia, artralgia, astenia e rash maculopapular, entre outros. Em alguns casos, pode levar à síndrome de Guillain-Barré, doença autoimune neurológica, que causa fraqueza muscular generalizada e paralisia (Lima-Camara, 2016). Além disso, o Brasil foi o primeiro país a identificar e confirmar uma possível relação entre infecção por ZIKV em mulheres grávidas e ocorrência de síndrome congênita do Zika nos recém-nascidos, que acarretarão custos diretos e indiretos ao longo de toda a vida (Araújo et al., 2017).

Quanto à prevenção das arboviroses, o Brasil conta com o Programa Nacional de Controle da Dengue, que tem como foco principal o controle do Aedes aegypti pela população e por profissionais, com apoio governamental, e com o Programa Nacional de Apoio ao Combate às Doenças Transmitidas pelo Aedes (Pronaedes), que tem como objetivo o financiamento de projetos de combate à proliferação das doenças transmitidas pelo vetor. No entanto, em vista do grande registro de casos de arboviroses nos últimos anos no Brasil, é possível concluir pela baixa efetividade do programa em reduzir as populações de vetores em níveis que poderiam interromper a transmissão das doenças (Araújo et al., 2015; Paes de Andrade et al., 2016).

Este estudo tem o objetivo de avaliar os custos de combate ao vetor, custos médicos diretos e custos indiretos associados à dengue clássica, à dengue hemorrágica, à chikungunya e à infecção pelo ZIKV no Brasil, para 2016.

\section{Métodos}

\section{Escopo da análise}

As análises quantificaram os gastos públicos relacionados ao combate ao vetor (repasse de recursos federais e aquisição de pesticidas), custos médicos diretos relacionados ao manejo das doenças no Sistema Único de Saúde (SUS) e custos indiretos relacionados ao absenteísmo, tanto na esfera pública quanto na privada.

Os resultados foram apresentados em escala nacional (Brasil) e segmentados por unidades federativas (UFs). 


\section{Custos de combate ao vetor}

Os custos relacionados ao combate às arboviroses, promovido pelo governo federal, foram definidos com base na Portaria no 1.378, que define as responsabilidades e diretrizes para execução e financiamento de ações de vigilância em saúde pela União, estados e municípios. A referida Portaria determina os recursos federais destinados ao componente de vigilância em saúde, que tem por função a vigilância, prevenção e controle de doenças e agravos (Ministério da Saúde [Brasil], 2013). Os recursos repassados mensalmente são constituídos pelo Piso Fixo de Vigilância em Saúde (PFVS), que determina uma verba fixa calculada com base na situação epidemiológica de cada estado e município, e pelo Piso Variável de Vigilância em Saúde (PVVS), que é determinado mediante adesão dos estados e municípios a programas específicos, regulamentado pelo Ministério da Saúde (MS) e tem por finalidade implantar e manter ações e serviços de vigilância em saúde, bem como programas de qualificação de pessoal. É importante ressaltar que se trata de verba destinada ao combate a vetores em geral e não exclusivamente destinada ao combate ao Aedes aegypti. Desta forma, assumiu-se que $65 \%$ do PFVS seria destinado ao combate às arboviroses (Felix, 2016; Ministério da Saúde [Brasil], 2013; Cambricoli, 2015). Esse valor foi testado em análise de sensibilidade. No caso do PVVS, como constitui um repasse adicional especificamente para combate ao Aedes aegypti, foi considerado $100 \%$ destinado a esse fim.

Os valores de repasse relacionados à manutenção das equipes de agentes de combate a endemias (ACEs) pelo governo federal foram definidos com base na assistência financeira complementar (AFC) fornecida mensalmente para complemento do piso salarial dos ACEs, bem como por um incentivo mensal repassado para o fortalecimento de políticas de combate a endemias.

Os valores de incentivo mensal e AFC, bem como os de PFVS e PVVS, foram obtidos da Portaria $n$ ㅇ․․129, de 28 de dezembro de 2016.

O custo de aquisição de inseticidas e larvicidas pelo governo federal foi obtido com base em valores fornecidos pelo próprio governo federal por meio da lei de acesso à informação (Ministério da Saúde [Brasil], 2016c).

\section{Custos médicos diretos}

O cálculo dos custos médicos diretos se deu por meio do estabelecimento de condutas de manejos ambulatorial e hospitalar das arboviroses.

O custo de tratamento ambulatorial dos episódios de dengue foi considerado de acordo com a estimativa de um estudo observacional que incluiu pacientes com dengue atendidos no SUS e no sistema suplementar, em quatro regiões brasileiras (Martelli et al., 2015). O estudo foi conduzido em 2013 e publicado em 2015, porém, como não houve atualização da tabela de reembolso ambulatorial do SUS desde então, o custo sob a perspectiva do SUS foi considerado sem ajuste pela inflação.

Para estimar o custo de tratamento ambulatorial dos episódios de chikungunya e Zika, foram estabelecidas condutas com base na literatura médica disponível (Ministério da Saúde [Brasil], 2017a; Ministério da Saúde [Brasil], 2016a; Ministério da Saúde [Brasil], 2016d; Federação Nacional de Saúde Suplementar, 2016; Secretaria Municipal de Saúde do Rio de Janeiro, 2016; Sociedade Brasileira de Reumatologia, 2016; Ministério da Saúde [Brasil], 2016e; Governo do Estado de São Paulo, 2015) e, posteriormente, quantificadas com base na monetização dos materiais e procedimentos envolvidos, por meio da técnica de microcosting, sob a perspectiva do SUS.

O custo de tratamento hospitalar dos episódios de dengue, chikungunya e Zika foi obtido do Sistema de Informações Hospitalares do Departamento de Informática do SUS (Datasus) para 2016 (Ministério da Saúde [Brasil], 2016f).

Após a quantificação desses custos, o custo anual total foi calculado por meio da multiplicação dos custos de manejo pelo número de casos notificados, sendo estes divididos em casos confirmados e suspeitos, quando disponível a informação.

Aos custos médicos diretos também foram somados os custos de manutenção dos centros especializados em reabilitação para as síndromes congênitas associadas ao ZIKV (Frasão et al., 2017). Em razão da falta de conhecimento atual sobre a evolução dos casos de pacientes com síndrome congênita do vírus Zika e dos pacientes com complicações neurológicas decorrentes do ZIKV (síndrome de Guillain-Barré, encefalites, mielite, entre outros), optou-se por não incluir esses custos de longo prazo no presente estudo.

\section{Custos indiretos}

No caso dos episódios de dengue, o custo indireto médio por paciente foi obtido do estudo de Martelli et al. (2015), relacionado aos períodos de afastamento do trabalho para recuperação dos pacientes (Martelli et al., 2015).

Para o cálculo dos custos indiretos relacionados aos episódios de Zika e chikungunya, foi necessário estimar os AVAls. Essa métrica define o tempo de vida perdido por um indivíduo como resultado de uma doença ou morte prematura e foi considerada para determinar o tempo, em anos, em que o indivíduo permanece afastado de sua atividade produtiva (Murray, 1994; Murray \& Lopez, 1994). Neste caso, para evitar a incerteza das estimativas de longo prazo, foram considerados somente os custos de curto prazo relacionados à perda de produtividade por afastamento do trabalho.

Os AVAls decorrentes da incidência de arboviroses foram calculados com base na equação 1 (Murray, 1994):

$$
A V A I=-\frac{D C e^{-b a}}{(b+r)^{2}}\left\{e^{-(b+r) L}[1+(b+r)(L+a)]-[1+(b+r) a]\right\}
$$

Equação 1: estimativa de AVAls. 
Em que $D$ representa o grau de incapacidade resultante da doença ( $D=1$ para morte e 0 para saúde perfeita); $C$ e $b$ representam os parâmetros de ajuste pela idade do paciente; a representa a idade de início da doença; $L$, a duração da doença até a recuperação total das funções produtivas do paciente ou o tempo de vida esperado perdido para a morte; r, uma variável de desconto do valor presente atribuída aos anos de vida futuros (o desconto assume que os anos vividos na juventude representam maior peso quando comparados àqueles em idade avançada, refletindo a perda da produtividade decorrente do envelhecimento) (Murray, 1994).

Os parâmetros referentes à função de ajuste por idade (C, b e r) foram obtidos do Global Burden of Disease Study (Murray \& Lopez, 1996). A duração e a idade de início da doença foram definidas com base na literatura relativa às arboviroses ou na base de dados do DATASUS (idade média de início da doença). Não foi considerada mortalidade nessa estimativa, portanto a taxa de mortalidade foi assumida como zero para focar somente nos custos indiretos de curto prazo (Tabela 1) (Instituto Brasileiro de Geografia e Estatística [IBGE], 2016).

Para os parâmetros idade média do paciente e duração da doença, que podem variar entre indivíduos, atribuiu-se uma distribuição de probabilidade uniforme para que valores aleatórios fossem gerados a cada simulação. O método de Monte Carlo foi usado para gerar mil iterações e os valores de AVAls foram calculados com base na média das simulações. Esse racional baseou-se no proposto por Luz et al. (2009), que estimou os AVAls por dengue para o estado do Rio de Janeiro (Luz et al., 2009).

A quantificação do custo relacionado à perda de produtividade foi realizada com base no PIB per capita nacional de R\$ 30.407, referente a 2016, publicado pelo IBGE (Instituto Brasileiro de Geografia e Estatística [IBGE], 2017) O cálculo dos custos indiretos baseou-se na equação 2:

Custos indiretos $=$ PIB per capita $\times$ Número de casos $\times$ AVAls

Equação 2: cálculo de custos indiretos

Tabela 1. Parâmetros do modelo de projeção de AVAls

\begin{tabular}{lcc}
\hline Parâmetro & Zika & Chikungunya \\
\hline Parâmetro de ajuste por idade - C & 0,16 & 0,16 \\
\hline Parâmetro de ajuste por idade - b & 0,04 & 0,04 \\
\hline Desconto - $r$ & $3 \%$ & $3 \%$ \\
\hline $\begin{array}{l}\text { Grau de comprometimento } \\
\text { da doença - D }\end{array}$ & 0,16 & $0,16-0,23$ \\
\hline Idade de início - a [ldade (DP)] & $31(6)$ & $41(7)$ \\
\hline Duração da doença - L (dias) & 8 & 14 \\
\hline
\end{tabular}

DP: desvio-padrão.

\section{Resultados}

\section{Custos de combate ao vetor}

Em 2016, o investimento total no combate ao vetor atingiu o patamar de aproximadamente $\mathrm{R} \$ 1,5$ bilhão no Brasil.

Os estados de São Paulo, Minas Gerais, Bahia e Rio de Janeiro receberam os maiores repasses federais para combate ao vetor, apresentando investimentos superiores a $R \$ 100$ milhões por estado em 2016. Tais resultados são esperados por se tratarem das UFs com maior população no País. No entanto, é importante notar que UFs com menor população, como Pará, Ceará, Pernambuco e Maranhão, integrantes das regiões Norte-Nordeste, vêm, em seguida, como as UFs a receber maior investimento em combate ao vetor no país (Tabela 2).

Por se tratar de um valor porcentual variável, a participação do PFVS destinada ao combate do Aedes aegypti foi testada em análise de sensibilidade, em um intervalo entre 60\% e 70\% (valor base de 65\%). Essa variação influenciou de maneira pouco significativa o investimento total no combate ao vetor, que variou entre R\$ 1,4 e R\$ 1,5 bilhão em todo o país.

O custo reportado pelo governo federal para aquisição de inseticidas e larvicidas em 2016 foi de R\$ 78,6 milhões.

\section{Custos médicos diretos}

O país apresentou um gasto total de aproximadamente R\$ 374 milhões no tratamento de arboviroses em 2016.

Minas Gerais, Bahia, São Paulo, Pernambuco e Rio de Janeiro são as cinco UFs que apresentaram maiores gastos relacionados ao tratamento das arboviroses no país (Tabela 3). Entre as dez principais UFs em gastos com o manejo das doenças, cinco estão localizadas na região Nordeste do País (Bahia, Ceará, Paraíba, Pernambuco e Rio Grande do Norte).

\section{Custos indiretos}

A febre chikungunya apresentou o maior número de AVAls perdidos por episódio da doença (0,036 AVAl), seguido pela infecção por ZIKV (0,005 AVAI).

Estima-se um custo indireto total de aproximadamente R\$ 431 milhões para o País com a perda de produtividade por afastamentos relacionados às arboviroses em 2016.

Minas Gerais, São Paulo e Bahia apresentaram os maiores custos indiretos relacionados às arboviroses, com valores que variaram entre $\mathrm{R} \$ 40$ milhões (BA) e R\$ 102 milhões (MG) (Tabela 4).

\section{Custos totais}

Considerando os custos totais com o manejo das arboviroses, o Brasil sofreu com um impacto de aproximadamente $R \$ 2,3$ bilhões em 2016.

Minas Gerais, São Paulo, Bahia e Rio de Janeiro apresentaram os maiores custos relacionados às arboviroses no Bra- 
Tabela 2. Custos relacionados ao combate ao vetor

\begin{tabular}{|c|c|c|c|c|c|}
\hline UF & $\begin{array}{c}\text { PFVS anual (65\% } \\
\text { do total) }\end{array}$ & AFC + incentivo anual & PVVS - Combate Aedes & Aquisição de pesticidas & Total \\
\hline Brasil & $\mathrm{R} \$ 584.215 .365$ & $\mathrm{R} \$ 655.989 .048$ & $\mathrm{R} \$ 152.103 .612$ & $\mathrm{R} \$ 78.682 .735$ & $\mathrm{R} \$ 1.470 .990 .760$ \\
\hline$A C$ & $\mathrm{R} \$ 4.396 .239$ & $R \$ 2.979 .132$ & $\mathrm{R} \$ 1.099 .412$ & $\mathrm{R} \$ 0$ & $\mathrm{R} \$ 8.474 .783$ \\
\hline$A L$ & $R \$ 9.310 .218$ & $R \$ 15.488 .850$ & $R \$ 3.337 .898$ & $\mathrm{R} \$ 0$ & $R \$ 28.136 .966$ \\
\hline AM & $R \$ 27.670 .866$ & $R \$ 4.877 .340$ & $R \$ 5.382 .734$ & $\mathrm{R} \$ 0$ & $R \$ 37.930 .940$ \\
\hline AP & $R \$ 3.808 .377$ & $R \$ 3.941 .418$ & $\mathrm{R} \$ 1.173 .274$ & $R \$ 0$ & $R \$ 8.923 .069$ \\
\hline BA & $R \$ 43.746 .542$ & $\mathrm{R} \$ 70.721 .430$ & $\mathrm{R} \$ 12.258 .327$ & $\mathrm{R} \$ 0$ & $R \$ 126.726 .299$ \\
\hline CE & $\mathrm{R} \$ 23.363 .120$ & $\mathrm{R} \$ 46.427 .004$ & $R \$ 7.520 .002$ & $\mathrm{R} \$ 0$ & $R \$ 77.310 .126$ \\
\hline DF & $R \$ 4.035 .343$ & $R \$ 6.116 .448$ & $R \$ 1.677 .922$ & $\mathrm{R} \$ 0$ & $\mathrm{R} \$ 11.829 .713$ \\
\hline ES & $R \$ 12.013 .152$ & $R \$ 12.733 .812$ & $R \$ 3.214 .791$ & $R \$ 0$ & $R \$ 27.961 .756$ \\
\hline GO & $\mathrm{R} \$ 17.710 .790$ & $R \$ 35.248 .668$ & $R \$ 5.791 .961$ & $\mathrm{R} \$ 0$ & $\mathrm{R} \$ 58.751 .420$ \\
\hline MA & $R \$ 33.214 .778$ & $R \$ 25.348 .986$ & $\mathrm{R} \$ 8.162 .039$ & $\mathrm{R} \$ 0$ & $R \$ 66.725 .802$ \\
\hline MG & $\mathrm{R} \$ 65.619 .464$ & $\mathrm{R} \$ 77.918 .802$ & $R \$ 16.715 .162$ & $\mathrm{R} \$ 0$ & $\mathrm{R} \$ 160.253 .427$ \\
\hline MS & $\mathrm{R} \$ 7.240 .381$ & $\mathrm{R} \$ 11.718 .798$ & $R \$ 2.142 .016$ & $R \$ 0$ & $\mathrm{R} \$ 21.101 .195$ \\
\hline MT & $R \$ 12.441 .670$ & $\mathrm{R} \$ 13.551 .096$ & $R \$ 3.085 .510$ & $R \$ 0$ & $\mathrm{R} \$ 29.078 .275$ \\
\hline PA & $R \$ 38.807 .558$ & $R \$ 32.651 .814$ & $\mathrm{R} \$ 11.414 .394$ & $R \$ 0$ & $\mathrm{R} \$ 82.873 .766$ \\
\hline PB & $\mathrm{R} \$ 13.504 .533$ & $\mathrm{R} \$ 14.368 .380$ & $\mathrm{R} \$ 2.876 .985$ & $\mathrm{R} \$ 0$ & $R \$ 30.749 .898$ \\
\hline PE & R\$ 28.288.781 & $R \$ 37.647 .792$ & $R \$ 6.966 .831$ & $\mathrm{R} \$ 0$ & $\mathrm{R} \$ 72.903 .404$ \\
\hline $\mathrm{PI}$ & $R \$ 9.333 .539$ & $R \$ 14.276 .106$ & $R \$ 2.650 .257$ & $\mathrm{R} \$ 0$ & R\$ 26.259.902 \\
\hline$P R$ & $R \$ 20.874 .131$ & $R \$ 33.970 .014$ & $\mathrm{R} \$ 4.991 .785$ & $\mathrm{R} \$ 0$ & $R \$ 59.835 .930$ \\
\hline RJ & $R \$ 52.792 .030$ & $R \$ 43.210 .596$ & $R \$ 13.443 .258$ & $\mathrm{R} \$ 0$ & $\mathrm{R} \$ 109.445 .884$ \\
\hline RN & $\mathrm{R} \$ 9.790 .003$ & $R \$ 18.204 .342$ & $\mathrm{R} \$ 2.753 .735$ & $\mathrm{R} \$ 0$ & $\mathrm{R} \$ 30.748 .079$ \\
\hline $\mathrm{RO}$ & R\$ 9.947.002 & $R \$ 4.152 .330$ & $\mathrm{R} \$ 2.291 .930$ & $\mathrm{R} \$ 0$ & R\$ 16.391.262 \\
\hline $\mathrm{RR}$ & $\mathrm{R} \$ 2.775 .691$ & $R \$ 3.453 .684$ & $R \$ 910.629$ & $\mathrm{R} \$ 0$ & $\mathrm{R} \$ 7.140 .004$ \\
\hline RS & $\mathrm{R} \$ 23.174 .554$ & $\mathrm{R} \$ 14.342 .016$ & $R \$ 5.218 .296$ & $\mathrm{R} \$ 0$ & $\mathrm{R} \$ 42.734 .866$ \\
\hline SC & $R \$ 14.220 .075$ & $R \$ 8.278 .296$ & $R \$ 3.214 .956$ & $R \$ 0$ & $\mathrm{R} \$ 25.713 .327$ \\
\hline SE & $R \$ 6.505 .828$ & $R \$ 9.873 .318$ & $\mathrm{R} \$ 1.728 .169$ & $\mathrm{R} \$ 0$ & $\mathrm{R} \$ 18.107 .314$ \\
\hline SP & $\mathrm{R} \$ 82.418 .397$ & R\$ 86.698.014 & $R \$ 20.306 .001$ & $\mathrm{R} \$ 0$ & R\$ 189.422 .412 \\
\hline TO & $R \$ 7.212 .304$ & $\mathrm{R} \$ 7.790 .562$ & $\mathrm{R} \$ 1.775 .337$ & $R \$ 0$ & $\mathrm{R} \$ 16.778 .204$ \\
\hline
\end{tabular}

UF: unidade federativa; PFVS: piso fixo de vigilância em saúde; PVVS: piso variável de vigilância em saúde.

sil. Pernambuco segue como a quinta maior UF em custos relacionados às doenças transmitidas pelo mosquito Aedes aegypti, em contraponto a sua população total, que chega a ser aproximadamente 50\% inferior à população da Bahia, UF que ocupa a terceira posição em gastos totais (Tabela 5).

\section{Discussão}

Neste trabalho foram estimados os custos globais envolvidos no manejo das arboviroses transmitidas pelo Aedes aegyptino Brasil e suas respectivas UFs, sob a perspectiva do pagador público. Foram estimados os custos relacionados ao combate ao vetor e custos médicos diretos envolvidos no tratamento das doenças (dengue clássica, dengue hemorrágica, chikungunya e infecção por ZIKV). Vale ressaltar que, embora a febre amarela seja uma arbovirose com impacto social e financeiro crescente no Brasil, não houve comprovação de que o Aedes aegypti tenha voltado a transmitir a doença. Por isso, os custos associados à vacinação contra febre amarela e ao tratamento dos casos confirmados da doença não foram incluídos no presente estudo.

Além disso, foram considerados os custos indiretos relacionados à perda de produtividade dos pacientes durante a infecção viral. O trabalho traz como inovação o cálculo do impacto social das principais arboviroses no País, representado pelos AVAls.

Estimou-se um custo total de aproximadamente $\mathrm{R} \$ 2,3$ bilhões em 2016, em que 65\%, 19\% e 16\% são representados por combate ao vetor, custos indiretos e custos médicos diretos, respectivamente. 
Tabela 3. Custos médicos diretos

\begin{tabular}{|c|c|c|c|c|}
\hline UF & Dengue & Outras arboviroses & $\begin{array}{c}\text { Manutenção dos centros } \\
\text { especializados em reabilitação }\end{array}$ & Total \\
\hline Brasil & $\mathrm{R} \$ 175.876 .163$ & $\mathrm{R} \$ 83.754 .369$ & $\mathrm{R} \$ 114.300 .000$ & $\mathrm{R} \$ 373.930 .532$ \\
\hline$A C$ & $R \$ 273.555$ & $R \$ 105.586$ & $R \$ 0$ & $R \$ 379.141$ \\
\hline $\mathrm{AL}$ & $R \$ 2.094 .841$ & $R \$ 4.821 .093$ & $\mathrm{R} \$ 0$ & $R \$ 6.915 .934$ \\
\hline AM & $R \$ 934.327$ & $\mathrm{R} \$ 525.100$ & $\mathrm{R} \$ 0$ & $R \$ 1.459 .426$ \\
\hline AP & $R \$ 206.829$ & $R \$ 265.133$ & $\mathrm{R} \$ 0$ & $R \$ 471.962$ \\
\hline BA & $R \$ 8.559 .568$ & $\mathrm{R} \$ 16.334 .531$ & $\mathrm{R} \$ 0$ & $\mathrm{R} \$ 24.894 .098$ \\
\hline CE & $R \$ 6.099 .629$ & $\mathrm{R} \$ 12.426 .695$ & $\mathrm{R} \$ 0$ & $\mathrm{R} \$ 18.526 .324$ \\
\hline DF & $\mathrm{R} \$ 2.161 .445$ & $\mathrm{R} \$ 177.127$ & $R \$ 0$ & $R \$ 2.338 .572$ \\
\hline ES & $R \$ 4.940 .563$ & $\mathrm{R} \$ 281.381$ & $\mathrm{R} \$ 0$ & $R \$ 5.221 .944$ \\
\hline GO & $\mathrm{R} \$ 14.190 .323$ & $R \$ 786.373$ & $\mathrm{R} \$ 0$ & $\mathrm{R} \$ 14.976 .695$ \\
\hline MA & $R \$ 3.468 .937$ & $R \$ 3.906 .428$ & $\mathrm{R} \$ 0$ & $R \$ 7.375 .365$ \\
\hline MG & $R \$ 60.169 .115$ & $\mathrm{R} \$ 1.371 .089$ & $\mathrm{R} \$ 0$ & $R \$ 61.540 .205$ \\
\hline MS & $R \$ 5.339 .118$ & $\mathrm{R} \$ 184.923$ & $\mathrm{R} \$ 0$ & $R \$ 5.524 .041$ \\
\hline MT & $\mathrm{R} \$ 2.317 .093$ & $R \$ 1.603 .698$ & $R \$ 0$ & $R \$ 3.920 .790$ \\
\hline PA & $R \$ 1.684 .053$ & $R \$ 1.384 .472$ & $\mathrm{R} \$ 0$ & $R \$ 3.068 .525$ \\
\hline PB & $R \$ 4.289 .618$ & $R \$ 5.340 .084$ & $R \$ 0$ & $R \$ 9.629 .701$ \\
\hline$P E$ & $R \$ 7.763 .251$ & $\mathrm{R} \$ 12.456 .924$ & $\mathrm{R} \$ 0$ & $R \$ 20.220 .175$ \\
\hline $\mathrm{PI}$ & $R \$ 691.529$ & $R \$ 723.978$ & $R \$ 0$ & $\mathrm{R} \$ 1.415 .508$ \\
\hline$P R$ & $\mathrm{R} \$ 7.531 .668$ & $\mathrm{R} \$ 302.381$ & $\mathrm{R} \$ 0$ & $\mathrm{R} \$ 7.834 .049$ \\
\hline RJ & $\mathrm{R} \$ 9.790 .978$ & $R \$ 9.108 .866$ & $\mathrm{R} \$ 0$ & R\$ 18.899 .844 \\
\hline RN & $R \$ 6.641 .743$ & $R \$ 6.594 .147$ & $\mathrm{R} \$ 0$ & $\mathrm{R} \$ 13.235 .890$ \\
\hline $\mathrm{RO}$ & $\mathrm{R} \$ 1.068 .897$ & $R \$ 270.932$ & $R \$ 0$ & $R \$ 1.339 .828$ \\
\hline RR & $\mathrm{R} \$ 53.653$ & $\mathrm{R} \$ 71.809$ & $\mathrm{R} \$ 0$ & $\mathrm{R} \$ 125.462$ \\
\hline RS & $R \$ 387.896$ & $\mathrm{R} \$ 101.671$ & $R \$ 0$ & $R \$ 489.567$ \\
\hline SC & $R \$ 646.861$ & $\mathrm{R} \$ 163.341$ & $\mathrm{R} \$ 0$ & $\mathrm{R} \$ 810.202$ \\
\hline SE & $\mathrm{R} \$ 419.833$ & $R \$ 2.349 .534$ & $R \$ 0$ & $R \$ 2.769 .368$ \\
\hline SP & $\mathrm{R} \$ 23.222 .769$ & $R \$ 1.596 .950$ & $\mathrm{R} \$ 0$ & $R \$ 24.819 .719$ \\
\hline TO & $\mathrm{R} \$ 928.072$ & $R \$ 500.124$ & $R \$ 0$ & $\mathrm{R} \$ 1.428 .196$ \\
\hline
\end{tabular}

Entre os custos relacionados ao combate ao vetor, aproximadamente $85 \%$ são representados pelo PFVS anual, direcionado ao combate ao Aedes aegypti, e manutenção de equipes de ACEs (incluindo o repasse do incentivo anual), com os repasses do PVVS e a aquisição de pesticidas representando apenas 10\% e 5\% do total, respectivamente. É importante ressaltar que o custo relacionado ao combate ao vetor (aproximadamente R\$ 1,5 bilhão) apresentado neste estudo é significativamente superior ao apresentado em outras publicações (Buen et al., 2017). Essa aparente incongruência está relacionada à inclusão do PFVS, estimado em $65 \%$ do PFVS total, dos custos de manutenção das equipes de ACEs e aquisição de pesticidas, além do repasse do PVVS. Considera-se esta uma abordagem mais realista, capaz de representar com mais fidedignidade os custos envolvidos no combate ao vetor no País.

Em contrapartida, não foram contemplados os investimentos diretos realizados pelos Estados e municípios no combate ao vetor. A forma como essas despesas são reportadas pelos Estados e municípios não permite diferenciar qual parte desse valor foi oriunda das transferências federais e qual parcela representa investimento direto das demais esferas de governo. Desta forma, para evitar o risco de dupla contagem dos investimentos federais, optou-se por não adicionar as despesas estaduais e municipais. A estimativa realizada de custos de combate ao vetor é, portanto, conservadora.

O tratamento dos pacientes infectados por arboviroses resultou em um custo total de aproximadamente R\$373 
Tabela 4. Custos indiretos

\begin{tabular}{|c|c|c|c|c|}
\hline UF & Dengue clássica & Chikungunya & Zika & Total \\
\hline Brasil & $\mathrm{R} \$ 293.341 .383$ & $\mathrm{R} \$ 123.943 .728$ & $\mathrm{R} \$ 13.986 .993$ & $\mathrm{R} \$ 431.272 .105$ \\
\hline$A C$ & $R \$ 459.451$ & $\mathrm{R} \$ 170.077$ & $R \$ 3.768$ & $R \$ 633.295$ \\
\hline$A L$ & $R \$ 3.422 .861$ & $R \$ 7.842 .693$ & $\mathrm{R} \$ 446.531$ & $\mathrm{R} \$ 11.712 .084$ \\
\hline AM & $R \$ 1.573 .654$ & $R \$ 403.534$ & $R \$ 291.863$ & $R \$ 2.269 .050$ \\
\hline AP & $\mathrm{R} \$ 347.736$ & $R \$ 423.597$ & $R \$ 29.362$ & $R \$ 800.694$ \\
\hline $\mathrm{BA}$ & $\mathrm{R} \$ 14.220 .362$ & $\mathrm{R} \$ 23.199 .780$ & $R \$ 3.362 .753$ & $R \$ 40.782 .895$ \\
\hline CE & $R \$ 10.199 .605$ & $R \$ 21.830 .500$ & $R \$ 279.650$ & $R \$ 32.309 .755$ \\
\hline DF & $R \$ 3.498 .863$ & $\mathrm{R} \$ 262.183$ & $\mathrm{R} \$ 23.191$ & $R \$ 3.784 .236$ \\
\hline ES & $R \$ 8.216 .845$ & $R \$ 207.923$ & $\mathrm{R} \$ 152.914$ & $R \$ 8.577 .682$ \\
\hline GO & $R \$ 23.829 .014$ & $\mathrm{R} \$ 179.196$ & $R \$ 668.887$ & $\mathrm{R} \$ 24.677 .098$ \\
\hline MA & $R \$ 5.717 .481$ & $R \$ 6.158 .794$ & $R \$ 293.811$ & $\mathrm{R} \$ 12.170 .086$ \\
\hline MG & $\mathrm{R} \$ 100.554 .066$ & $R \$ 652.494$ & $R \$ 937.754$ & $\mathrm{R} \$ 102.144 .314$ \\
\hline MS & $R \$ 8.925 .925$ & $\mathrm{R} \$ 124.936$ & $\mathrm{R} \$ 111.665$ & $R \$ 9.162 .526$ \\
\hline MT & $R \$ 3.878 .343$ & $R \$ 254.432$ & $R \$ 1.423 .325$ & $R \$ 5.556 .100$ \\
\hline PA & $R \$ 2.738 .608$ & $\mathrm{R} \$ 1.792 .876$ & $R \$ 293.876$ & $R \$ 4.825 .360$ \\
\hline PB & $R \$ 7.187 .529$ & $R \$ 9.110 .747$ & $R \$ 243.598$ & $R \$ 16.541 .874$ \\
\hline PE & $R \$ 12.858 .652$ & $R \$ 22.257 .745$ & $R \$ 25.399$ & $R \$ 35.141 .795$ \\
\hline $\mathrm{Pl}$ & $R \$ 1.151 .084$ & $R \$ 1.255 .743$ & $R \$ 15.136$ & $R \$ 2.421 .963$ \\
\hline PR & $\mathrm{R} \$ 12.530 .520$ & $\mathrm{R} \$ 444.571$ & $\mathrm{R} \$ 46.381$ & $R \$ 13.021 .472$ \\
\hline RJ & $R \$ 16.347 .490$ & $R \$ 8.156 .401$ & $\mathrm{R} \$ 4.452 .447$ & $\mathrm{R} \$ 28.956 .337$ \\
\hline RN & $\mathrm{R} \$ 11.198 .357$ & $\mathrm{R} \$ 11.350 .018$ & $R \$ 256.785$ & $\mathrm{R} \$ 22.805 .160$ \\
\hline $\mathrm{RO}$ & $\mathrm{R} \$ 1.765 .627$ & $R \$ 366.600$ & $\mathrm{R} \$ 63.400$ & $R \$ 2.195 .628$ \\
\hline $\mathrm{RR}$ & $\mathrm{R} \$ 59.340$ & $R \$ 108.065$ & $\mathrm{R} \$ 11.563$ & R\$ 178.968 \\
\hline RS & $R \$ 648.232$ & $\mathrm{R} \$ 154.574$ & $\mathrm{R} \$ 12.147$ & $\mathrm{R} \$ 814.954$ \\
\hline SC & $\mathrm{R} \$ 1.058 .759$ & $R \$ 259.903$ & $R \$ 5.976$ & $R \$ 1.324 .639$ \\
\hline SE & $R \$ 681.691$ & $R \$ 4.209 .064$ & $\mathrm{R} \$ 14.291$ & $R \$ 4.905 .046$ \\
\hline SP & $\mathrm{R} \$ 38.711 .974$ & $\mathrm{R} \$ 2.144 .429$ & $R \$ 371.633$ & $R \$ 41.228 .036$ \\
\hline $\mathrm{TO}$ & $R \$ 1.559 .315$ & $R \$ 622.856$ & $\mathrm{R} \$ 148.887$ & $R \$ 2.331 .058$ \\
\hline
\end{tabular}

milhões. Deste, aproximadamente R\$ 176 milhões foram destinados ao manejo das infecções pelo DENV (clássica e hemorrágica). As outras arboviroses, juntas, representam um total de R\$ 84 milhões em custos médicos diretos. Os custos destinados ao tratamento das infecções por DENV representam $47 \%$ dos custos médicos diretos totais, enquanto as outras arboviroses juntas, apenas 22\%. Esse achado está de acordo com o esperado, uma vez que as infecções pelo DENV são as mais incidentes no Brasil, entre as arboviroses. Os valores estimados para o tratamento das infecções pelo DENV neste trabalho (R\$ 176 milhões) se contrapõem aos valores estimados por Martelli et al. (2015) que totalizaram aproximadamente R\$372 milhões (US\$ 164 milhões em 2013; R\$ $1=$ US\$ 0,44079) em custos médicos diretos para o trata- mento da dengue (Martelli et al., 2015). Essa diferença entre os resultados é determinada principalmente pelo volume de atendimentos ambulatoriais e hospitalizações por infecções pelo DENV considerados em cada análise. Nosso estudo considerou que 75\% dos casos de dengue reportados em 2016 seriam atendidos pelo SUS, totalizando 1,12 milhão de episódios de DENV atendidos no SUS, sendo aproximadamente 61 mil em ambiente hospitalar. O estudo de Martelli et al. (2015) considerou 2 milhões de episódios de DENV em 2013, sendo 201 mil atendidos em ambiente hospitalar. A diferença na proporção de casos atendidos em ambiente hospitalar se deu porque no presente estudo foram considerados os volumes de hospitalizações extraídos do DATASUS, enquanto o estudo de Martelli et al. (2015) aplicou a proporção de 
Tabela 5. Custos totais

\begin{tabular}{|c|c|c|c|c|}
\hline UF & Combate ao vetor & Custos médicos diretos & Custos indiretos & Total \\
\hline Brasil & $\mathrm{R} \$ 1.470 .990 .760$ & $\mathrm{R} \$ 373.930 .532$ & $\mathrm{R} \$ 431.272 .105$ & $\mathrm{R} \$ 2.276 .193 .396$ \\
\hline$A C$ & $\mathrm{R} \$ 8.474 .783$ & $R \$ 379.141$ & $R \$ 633.295$ & $R \$ 9.487 .220$ \\
\hline$A L$ & $\mathrm{R} \$ 28.136 .966$ & $R \$ 6.915 .934$ & $\mathrm{R} \$ 11.712 .084$ & $\mathrm{R} \$ 46.764 .985$ \\
\hline AM & $R \$ 37.930 .940$ & $\mathrm{R} \$ 1.459 .426$ & $R \$ 2.269 .050$ & $R \$ 41.659 .417$ \\
\hline AP & $R \$ 8.923 .069$ & $R \$ 471.962$ & $\mathrm{R} \$ 800.694$ & $R \$ 10.195 .725$ \\
\hline BA & $\mathrm{R} \$ 126.726 .299$ & $R \$ 24.894 .098$ & $R \$ 40.782 .895$ & R\$ 192.403 .292 \\
\hline CE & $R \$ 77.310 .126$ & $\mathrm{R} \$ 18.526 .324$ & $R \$ 32.309 .755$ & $R \$ 128.146 .205$ \\
\hline DF & $\mathrm{R} \$ 11.829 .713$ & $\mathrm{R} \$ 2.338 .572$ & $R \$ 3.784 .236$ & $R \$ 17.952 .522$ \\
\hline ES & $R \$ 27.961 .756$ & $\mathrm{R} \$ 5.221 .944$ & $\mathrm{R} \$ 8.577 .682$ & $R \$ 41.761 .381$ \\
\hline GO & $R \$ 58.751 .420$ & $R \$ 14.976 .695$ & $R \$ 24.677 .098$ & $R \$ 98.405 .212$ \\
\hline MA & $R \$ 66.725 .802$ & R\$ 7.375 .365 & $R \$ 12.170 .086$ & $R \$ 86.271 .252$ \\
\hline MG & $R \$ 160.253 .427$ & $R \$ 61.540 .205$ & $\mathrm{R} \$ 102.144 .314$ & $R \$ 323.937 .946$ \\
\hline MS & $\mathrm{R} \$ 21.101 .195$ & $\mathrm{R} \$ 5.524 .041$ & $R \$ 9.162 .526$ & $R \$ 35.787 .763$ \\
\hline MT & $R \$ 29.078 .275$ & $\mathrm{R} \$ 3.920 .790$ & $\mathrm{R} \$ 5.556 .100$ & $R \$ 38.555 .166$ \\
\hline PA & $\mathrm{R} \$ 82.873 .766$ & $R \$ 3.068 .525$ & $R \$ 4.825 .360$ & $\mathrm{R} \$ 90.767 .651$ \\
\hline PB & $\mathrm{R} \$ 30.749 .898$ & $R \$ 9.629 .701$ & $\mathrm{R} \$ 16.541 .874$ & $\mathrm{R} \$ 56.921 .474$ \\
\hline PE & $R \$ 72.903 .404$ & $R \$ 20.220 .175$ & $R \$ 35.141 .795$ & $R \$ 128.265 .375$ \\
\hline $\mathrm{PI}$ & $R \$ 26.259 .902$ & $\mathrm{R} \$ 1.415 .508$ & $\mathrm{R} \$ 2.421 .963$ & $\mathrm{R} \$ 30.097 .373$ \\
\hline$P R$ & $R \$ 59.835 .930$ & $R \$ 7.834 .049$ & $R \$ 13.021 .472$ & $R \$ 80.691 .450$ \\
\hline RJ & $\mathrm{R} \$ 109.445 .884$ & $R \$ 18.899 .844$ & $\mathrm{R} \$ 28.956 .337$ & $\mathrm{R} \$ 157.302 .065$ \\
\hline RN & $R \$ 30.748 .079$ & $R \$ 13.235 .890$ & $R \$ 22.805 .160$ & $R \$ 66.789 .130$ \\
\hline $\mathrm{RO}$ & $\mathrm{R} \$ 16.391 .262$ & R\$ 1.339.828 & $\mathrm{R} \$ 2.195 .628$ & $R \$ 19.926 .718$ \\
\hline RR & $R \$ 7.140 .004$ & $\mathrm{R} \$ 125.462$ & $\mathrm{R} \$ 178.968$ & $\mathrm{R} \$ 7.444 .434$ \\
\hline RS & $R \$ 42.734 .866$ & $R \$ 489.567$ & $\mathrm{R} \$ 814.954$ & $\mathrm{R} \$ 44.039 .387$ \\
\hline SC & $R \$ 25.713 .327$ & $\mathrm{R} \$ 810.202$ & $\mathrm{R} \$ 1.324 .639$ & $R \$ 27.848 .167$ \\
\hline SE & R\$ 18.107.314 & $R \$ 2.769 .368$ & $\mathrm{R} \$ 4.905 .046$ & $\mathrm{R} \$ 25.781 .728$ \\
\hline SP & $\mathrm{R} \$ 189.422 .412$ & $\mathrm{R} \$ 24.819 .719$ & $\mathrm{R} \$ 41.228 .036$ & $R \$ 255.470 .166$ \\
\hline TO & $\mathrm{R} \$ 16.778 .204$ & $R \$ 1.428 .196$ & $R \$ 2.331 .058$ & $R \$ 20.537 .458$ \\
\hline
\end{tabular}

$\mathrm{R} \$ 600.000 .000$

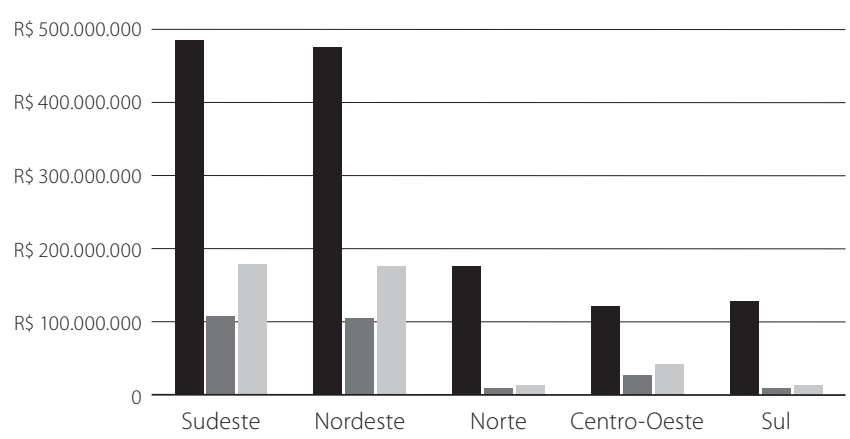

Combate ao vetor

Custos médicos diretos

Custos indiretos

Figura 1. Segmentação de custos por região. casos atendidos em hospitais observada na amostra do estudo para estimar o total de pacientes atendidos em ambiente hospitalar no SUS (Martelli et al., 2015).

Por tratar-se da perspectiva do pagador público, optou-se pela utilização dos custos repassados pelo governo federal para o manejo dos casos de dengue. Desta forma, os custos de hospitalização foram extraídos diretamente do DATASUS, com base na classificação CID-10 inserida no sistema, estando, assim, sujeitos a falhas na notificação, caracterizando-se como uma possível limitação deste estudo.

Foram ainda incluídos nesse montante os valores destinados à manutenção dos centros especializados de reabilitação (R\$ 114 milhões), destinados ao tratamento de pacientes afetados pelas complicações neurológicas atribuí- 
das às arboviroses. Vale ressaltar que os demais custos médicos diretos relacionados ao tratamento dos pacientes com complicações neurológicas associadas às infecções pelo ZIKV e complicações de longo prazo decorrentes das limitações impostas pela ocorrência de síndrome congênita do vírus Zika não foram contemplados na análise em razão das incertezas existentes quanto à história natural dessas doenças e, consequentemente, do uso de recursos e custos envolvidos.

Os custos indiretos, estimados com base em dados publicados e nos AVAls e PIB per capita nacional, resultaram em um total de aproximadamente R\$ 431 milhões em 2016. As infecções causadas pelo DENV representam 68\% do valor total, já a febre chikungunya, 29\%. Das arboviroses, a dengue apresentou o maior impacto em perda de produtividade. Esse resultado está alinhado com o apresentado por Luz et al. (2009) e segue o de outros países da América Latina (Gubler \& Meltzer, 1999; Luz et al., 2009).

Em termos gerais, os resultados para as UFs seguiram o mesmo padrão apresentado no âmbito nacional. Um ponto de destaque entre os resultados por UF é o elevado valor empenhado para o manejo da doença (custos médicos diretos) nas UFs pertencentes à região Nordeste, representando 40\% dos custos médicos diretos totais (R\$ 105 milhões) (Figura 1). Esse valor é semelhante ao apresentado pela região Sudeste (42\%).

Já em relação aos custos de combate ao vetor, as regiões Sudeste e Nordeste apresentam juntas aproximadamente 70\% do valor total destinado a essa finalidade, com 35\% (R\$ 487 miIhões) e 34\% (R\$ 478 milhões), respectivamente (Figura 1).

A região Sudeste apresentou a maior parcela de perdas relacionadas ao absenteísmo por arboviroses, $\mathrm{R} \$ 181$ milhões (42\% do valor total) (Figura 1).

Entre as limitações do estudo, podem ser citados: o foco em custos de combate ao vetor da esfera federal, desconsiderando investimentos diretos estaduais e municipais; a estimativa dos custos de manejo dos episódios de chikungunya e Zika por microcosting, diante da falta de estudos publicados sobre o tema; o risco de subapontamento dos casos de arboviroses tratados em ambiente hospitalar no SUS e registrados no DATASUS, o que levaria a uma subestimativa dos custos de tratamento hospitalar dos episódios de arboviroses; e o fato de não terem sido incluídos custos relacionados ao tratamento das complicações neurológicas associadas ao ZIKV e dos pacientes que evoluem com a síndrome congênita do $Z I K V$, diante das incertezas relacionadas à história natural das doenças.

\section{Conclusão}

As arboviroses são responsáveis por um considerável impacto econômico e social ao Brasil. Os custos relacionados ao seu manejo, entre combate ao vetor, custos médicos diretos e custos indiretos, representaram, juntos, aproximadamente 2\% do orçamento previsto para a saúde, no País, em 2016. O impacto à sociedade é relevante, com redução de aproximadamente R\$ 431 milhões ao ano associada à perda de produtividade decorrente das arboviroses.

\section{Referências bibliográficas}

Araújo HRC, Carvalho DO, loshino RS, Costa-da-Silva AL, Capurro ML. Aedes aegypti control strategies in Brazil: incorporation of new technologies to overcome the persistence of dengue epidemics. Insects. 2015;6(2):576-94.

Araújo TVB, Ximenes RA de A, Miranda-Filho D de B, Souza WV, Montarroyos UR, de Melo APL, et al. Association between microcephaly, Zika virus infection, and other risk factors in Brazil: final report of a case-control study. Lancet Infect Dis. 2017;3099(17):1-9.

Buen C, Almeida P, Castro A, Retamero A, Clark L. Aedes Aegypti: economic impact of prevention versus palliation of diseases caused by the mosquito. 6th ISPOR Latin America Conference, 2017.

Cambricoli F. Em 5 anos, dengue custa R\$ 4,2 bilhões à União [Internet]. Estadão. 2015. Disponível em: http://saude.estadao.com.br/noticias/ geral,em-5-anos--dengue-custa-r-4-2-bilhoes-a-uniao,1701794.

Donalisio MR, Freitas ARR, Zuben APB Von. Arboviroses emergentes no Brasil: desafios para a clínica e implicações para a saúde pública. Rev Saúde Pública. 2017;31(30):10-5.

Federação Nacional de Saúde Suplementar. Guia Zika, dengue e Chikungunya. 2016.

Felix L. Falta transparência nos gastos para o combate à dengue [Internet]. Gazeta do Povo. 2016. Disponível em: http://www.gazetadopovo.com. br/vida-e-cidadania/falta-transparencia-nos-gastos-para-o-combate-adengue-db6q5u0ahuzs6dy07uxg3pqdy

Frasão G, Maciel V, Bogaz C. Mato Grosso do Sul ganha reforço de R\$ 5,8 milhões para reabilitar crianças [Internet]. Portal da Saúde SUS. 2017. Disponível em: http://portalsaude.saude.gov.br/index.php/cidadao/ principal/agencia-saude/27967-mato-grosso-do-sul-ganha-reforco-der-5-8-milhoes-para-reabilitar-criancas

Gould E, Pettersson J, Higgs S, Charrel R, de Lamballerie X. Emerging arboviruses: why today? One Heal. 2017;4(June):1-13.

Governo do Estado de São Paulo. Secretaria de Saúde. Manejo clínico de paciente com suspeita de dengue. Guia prático. São Paulo; 2015.

Gregianini TS, Ranieri T, Favreto C, Nunes ZMA, Tumioto Giannini GL, Sanberg ND, et al. Emerging arboviruses in Rio Grande do Sul, Brazil: Chikungunya and Zika outbreaks, 2014-2016. Rev Med Virol. 2017;(August):1-10

Gubler DJ, Meltzer M. Impact of dengue/dengue hemorrhagic fever on the developing world. Adv Virus Res. 1999;53:35-70.

Instituto Brasileiro de Geografia e Estatística (IBGE). Diretoria de Pesquisas. Coordenação de População e Indicadores Sociais. Tábua completa de mortalidade para o Brasil - 2015: breve análise da evolução da mortalidade no Brasil. Rio de Janeiro: IBGE; 2016. 26 p.

Instituto Brasileiro de Geografia e Estatística (IBGE). PIB - Per capita - Brasil - 2010/2016 [Internet]. Brasil em síntase. 2017. Disponível em: https:// brasilemsintese.ibge.gov.br/contas-nacionais/pib-per-capita.

Lima-Camara TN. Emerging arboviruses and public health challenges in Brazil. Rev Saude Publica. 2016;50:1-7.

Luz PM, Grinsztejn B, Galvani AP. Disability adjusted life years lost to dengue in Brazil. Trop Med Int Heal. 2009;14(2):237-46. 
Martelli CMT, Siqueira JB, Parente MPPD, Zara AL de SA, Oliveira CS, Braga $C$, et al. Economic impact of dengue: multicenter study across four Brazilian regions. PLoS Negl Trop Dis. 2015;9(9):e0004042.

Minisério da Saúde (Brasil). Secretaria de Vigilância em Saúde. Departamento de Vigilância das Doenças Transmissíveis. 5. ed. Dengue: diagnóstico e manejo clínico adulto e criança. Brasília; 2016a.

Ministério da Saúde (Brasil). Portaria n-1.378, de 9 de julho de 2013: Regulamenta as responsabilidades e define diretrizes para execução e financiamento das ações de Vigilância em Saúde pela União, Estados, Distrito Federal e Municípios, relativos ao Sistema Nacional de Vigilância e [Internet]. 2013. Disponível em: http://bvsms.saude.gov.br/bvs/ saudelegis/gm/2013/prt1378_09_07_2013.html

Ministério da Saúde (Brasil). Secretaria de Vigilância em Saúde. Monitoramento dos casos de dengue, febre de chikungunya e febre pelo vírus Zika até a Semana Epidemiológica 52, 2016. Bol Epidemiológico. 2017b;48(3).

Ministério da Saúde (Brasil). Aquisição de inseticidas para uso em Saúde Pública [Internet]. Portal de Dados Abertos sobre Agrotóxicos. 2016c. Disponível em: http://dados.contraosagrotoxicos.org/dataset/aquisicaode-inseticidas-para-uso-em-saude-publica

Ministério da Saúde (Brasil). Zica: abordagem clínica na atenção básica. 2016e.

Ministério da Saúde (Brasil). Departamento de Informática do SUS (DATASUS). Informações de Saúde (TABNET) [Internet]. 2016f. Disponível em: http:// www2.datasus.gov.br/DATASUS/index.php?area=0205\&VDbj=http:/

Ministério da Saúde (Brasil). Secretaria de Atenção à Saúde. Diretrizes de estimulação precoce: crianças de zero a 3 anos com atraso no desenvolvimento neuropsicomotor decorrente de microcefalia. Brasília; 2016d.
Ministério da Saúde (Brasil). Secretaria de Vigilância em Saúde. Chikungunya: manejo clínico. Brasília; 2017a.

Ministério da Saúde (Brasil) Secretaria de Vigilância em Saúde. Monitoramento dos casos de dengue, febre de chikungunya e febre pelo vírus Zika até a Semana Epidemiológica 52, 2015. Bol Epidemiológico. 2016b;47(3):1-7.

Murray CJL. Quantifying the burden of disease: the technical basis for disability-adjusted life years. Bull World Health Organ. 1994;72(3):429-45.

Murray CJL, Lopez AD. Quantifying disability: data, methods and results. Bull World Health Organ. 1994;72(3):481-94.

Murray C, Lopez A. The global burden of disease. Cambridge: Harvard University Press; 1996.

Nunes MRT, Faria NR, de Vasconcelos JM, Golding N, Kraemer MU, de Oliveira $L F$, et al. Emergence and potential for spread of Chikungunya virus in Brazil. BMC Med. 2015;13(1):102.

Paes de Andrade P, Aragão FJL, Colli W, Dellagostin OA, Finardi-Filho F, Hirata $\mathrm{MH}$, et al. Use of transgenic Aedes aegypti in Brazil: risk perception and assessment. Bull World Health Organ. 2016;94(10):766-71.

Secretaria Municipal de Saúde do Rio de Janeiro. Subsecretaria Atenção Hospitalar Urgência e Emergência. Subsecretaria de Promoção Atenção Primária e Vigilância em Saúde. Protocolo de Chikungunya: assistência e vigilância [Internet]. Rio de Janeiro; 2016.

Shepard DS, Coudeville L, Halasa YA, Zambrano B, Dayan GH. Economic impact of dengue illness in the Americas. Am J Trop Med Hyg. 2011;84(2):200-7.

Sociedade Brasileira de Reumatologia. Recomendações da Sociedade Brasileira de Reumatologia para diagnóstico e tratamento da febre Chikungunya. 2016. 